\title{
The Evolutionary Properties on Solitary Solutions of Nonlinear Evolution Equations
}

\author{
Wenxia Chen, Danping Ding, Xiaoyan Deng, and Gang Xu \\ Nonlinear Scientific Research Center, Jiangsu University, Zhenjiang, Jiangsu 212013, China \\ Correspondence should be addressed to Gang Xu; gxu@ujs.edu.cn
}

Received 12 June 2017; Revised 19 September 2017; Accepted 28 September 2017; Published 29 October 2017

Academic Editor: Andrei D. Mironov

Copyright (c) 2017 Wenxia Chen et al. This is an open access article distributed under the Creative Commons Attribution License, which permits unrestricted use, distribution, and reproduction in any medium, provided the original work is properly cited.

The evolution process of four class soliton solutions is investigated by basic calculus theory. For any given $x$, we describe the special curvature evolution following time $t$ for the curve of soliton solution and also study the fluctuation of solution curve.

\section{Introduction}

Engineer J. Scott Russel is always mentioned when one talks about solitary phenomenon, because he is the first person who discovered the solitary phenomenon and reported it. Korteweg and de Vries constructed partial differential equation, which named KdV equation, to describe such solitary phenomenon. Kruskal and Zabusky proved that the solitary solutions of $\mathrm{KdV}$ equation possessed the particle property, and they firstly created the terminology "soliton" in 1965. Their works aroused an upsurge of study on solitary. In 1993, Camassa and Holm proposed a new model on shallow water wave motion, named as Camassa-Holm equation $(\mathrm{CH}$ equation). The significant property of $\mathrm{CH}$ equation is that the equation has a special weak solution with solitary and a peak at point $x=c t$, where $c$ is the wave propagation speed (see [1]). Constantin and Strauss proved the stability on peak solitary solution of the $\mathrm{CH}$ equation (see [2]). These different solitaries such as the smooth solitary of $\mathrm{KdV}$ equation and peakon of $\mathrm{CH}$ equation attracted attention which comes from different research areas, such as mathematics, physics, communication technology, image process, and artificial intelligence. In recent twenty years, there are tremendous amount of works about solitary solutions, and more types of solitary are established such as compact soliton, kink soliton, and bright and dark soliton. Soliton theory and application have formed interesting great mass fervor of nonlinear partial differential equation (see [1,3-5]).

On the other hand, the profiles of plane curves or intersurface attracted more and more attention. There have a mount of works about curvature motion and curvature motion flow (see [6-11]). Particularly, the curve evolution problem played important role in applied sciences. The study of immersed closed curves, which are introduced from their curvature functions, can apply to crystal growth (see $[12,13]$ ), flame propagation (see $[14,15]$ ), and curve shortening (see $[16,17])$. And also the bound for the evolution of length and curvature was investigated by [18]. Inspirited by above works, here we are interested in the evolution about some geometrical characteristics for the solutions of evolution partial differential equations with initial data; especially, we focus on the development of geometric properties of initial curve. This paper shall review some solitary solutions in view of evolution which arise from the $\mathrm{KdV}$ and modified $\mathrm{KdV}$ equation, $\mathrm{CH}$ equation, and Sine-Gordon equation.

The reminder of this paper is organized as follows. In Section 2, we introduce some basic notations and concepts which will be used in the following section. In Section 3, we investigate the evolution process of four class solitary solutions.

\section{Notations}

Suppose $u(x, t)$ be a solution of partial differential equation with initial data $u(x, 0)=u_{0}(x)$, where $x \in R$ is a spatial variable and $t>0$ is a time variable; we consider plane curve $\Gamma_{t}=\{(x, u(x, t))\}$, then the curvature of the plane curve is defined as

$$
K(t, x)=\frac{u_{x x}}{\left(1+u_{x}^{2}\right)^{3 / 2}},
$$


and the sign of the curvature indicates the orientation of curve bending.

Introducing a cumulative curvature of the curve $\Gamma_{t}$ over interval $(-\infty, x)$ as

$$
I(t, x)=\int_{-\infty}^{x} K(t, x) d x
$$

by computation, we have

$$
I(t, x)=\frac{u_{x}}{\sqrt{1+u_{x}^{2}}} .
$$

And denote the length of the curve by $g(t, x)$, that is, $g(t, x)=$ $\left(1+u_{x}^{2}\right)^{1 / 2}$. Thus, we obtain

$$
\begin{aligned}
& \frac{d K(t, x)}{d t} \\
& =\frac{1}{g^{3}(t, x)}\left[u_{x x t}-3 K(t, x) g^{2}(t, x) \frac{d g(t, x)}{d t}\right], \\
& \frac{d I(t, x)}{d t}=\frac{u_{x t}}{g^{3}(t, x)} .
\end{aligned}
$$

We will investigate these evolutional properties of solitary solutions as follows:

(1) $\mathrm{KdV}$ equation

$$
u_{t}+u u_{x}+\mu u_{x x x}=0
$$

as in [5], it is easy to check that it has solitary solution

$$
u(t, x)=u(x-D t)=3 D \operatorname{sech}^{2} \frac{\sqrt{D}}{2}(x-D t),
$$

where $D$ is a constant.

(2) Modified KdV equation

$$
u_{t}-6 u^{2} u_{x}+u_{x x x}=0
$$

as in [5], it has kink solution

$$
u(t, x)=u(x+D t)= \pm \sqrt{\frac{D}{2}} \tanh \sqrt{\frac{D}{2}}(x+D t)
$$

where $D$ is a constant.

(3) Sine-Gordon equation

$$
u_{t t}-u_{x x}=\sin u
$$

as in [19], it has bright an dark soliton solution

$$
u(t, x)=u(x-D t)=\arctan \frac{\sqrt{1-D^{2}}}{x-D t}
$$

where $D$ is a constant.
(4) Camassa-Holm equation

$$
u_{t}-u_{x x t}+3 u u_{x}=2 u_{x} u_{x x}+u u_{x x x}
$$

as in [1], it has peakon solution

$$
u(t, x)=c e^{-|x-c t|},
$$

where $c$ is a constant.

Considering $\Gamma_{t}=\{(x, u(x, t))\}$ as a family of single parametric curve with parameter $t$, we shall investigate the evolution procedure of some properties of parametric curve $\Gamma_{t}$, such as monotonicity, extremum, and curvature.

\section{Evolution Procedure of Solitary Solution}

3.1. $K d V$ Equation. In order to compute $K(t, x)$ and $I(t, x)$, firstly we need to calculate $u_{x}(x, t)$. And it follows from expression (6) that

$$
\begin{aligned}
& u_{x}(t, x)=-3 D^{3 / 2} \operatorname{sech}^{2} \xi \tanh \xi, \\
& \text { where } \xi=\frac{\sqrt{D}}{2}(x-D t) .
\end{aligned}
$$

Take a derivative (13) with respect to $t$,

$$
u_{x t}=\frac{3 D^{3}}{2} \operatorname{sech}^{2} \xi\left(3 \operatorname{sech}^{2} \xi-2\right) .
$$

Take a derivative (13) with respect to $x$,

$$
u_{x x}=\frac{3 D^{2}}{2} \operatorname{sech}^{2} \xi\left(2-3 \operatorname{sech}^{2} \xi\right) .
$$

Through above formulas, we obtain

$$
I(t, x)=-\frac{3 D^{3 / 2}}{g} \operatorname{sech}^{2} \xi \tanh \xi
$$

By differential (16) with respect to $t$,

$$
\frac{d I}{d t}=\frac{3}{2}\left(\frac{D}{g}\right)^{3} \operatorname{sech}^{2} \xi\left(3 \operatorname{sech}^{2} \xi-2\right) .
$$

And it is follows from the definition of $K(t, x)$ that

$$
\begin{aligned}
K(t, x) & =\frac{3}{2 D}\left(\frac{D}{g}\right)^{3} \operatorname{sech}^{2} \xi\left(2-3 \operatorname{sech}^{2} \xi\right), \\
\frac{d K(t, x)}{d t} & =\frac{3 D^{7}}{4 g^{5}} A \operatorname{sech}^{2} \xi \tanh \xi,
\end{aligned}
$$

where $A=A(\xi)=4-12 \operatorname{sech}^{2} \xi-72 D^{3} \operatorname{sech}^{4} \xi+216 D^{3} \operatorname{sech}^{6} \xi-$ $135 D^{3} \operatorname{sech}^{8} \xi$.

From calculus theory, we know that the extremum point from $\Gamma_{0}$ at $x=0$ develops to $\Gamma_{t}$ at $x=D t$ and the inflection point of $\Gamma_{0}$ from $(\ln ((\sqrt{6} \pm \sqrt{2}) / 2), 2 D)$ develops to $(D t+$ $\ln ((\sqrt{6} \pm \sqrt{2}) / 2), 2 D)$. It is well known that the inflection 


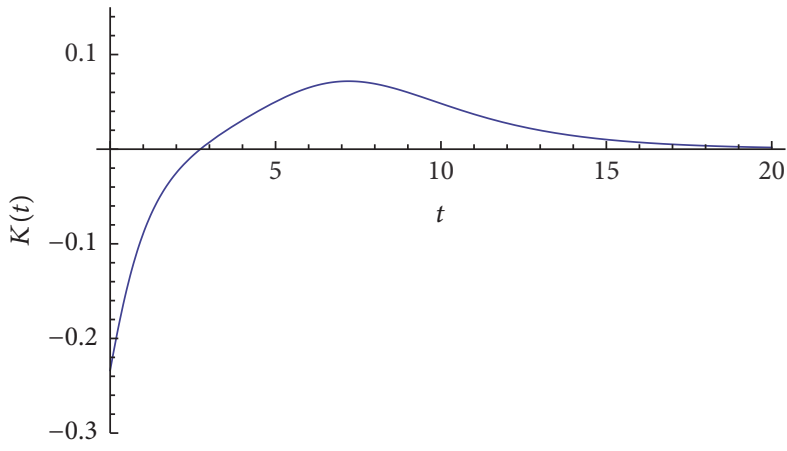

(a) $x=-0.5$

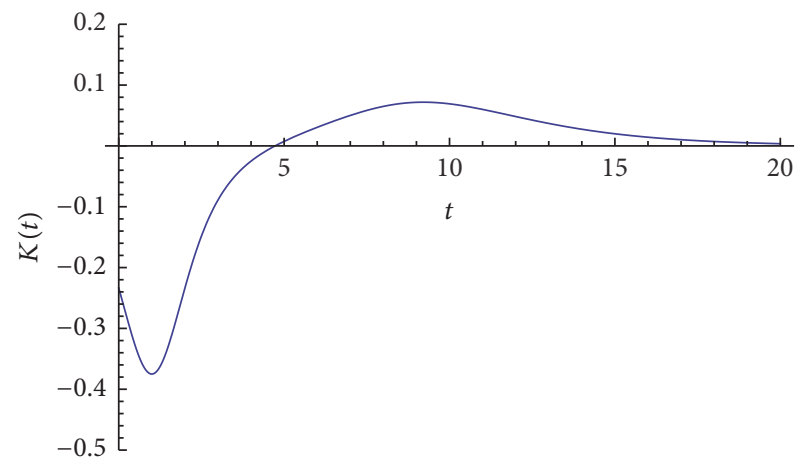

(b) $x=0.5$

Figure 1: The curvature $K(t, x)$ for different given $x$.

point can change the sign of curvature $K(t, x)$. For any fixed $x \in R$, the extremum will arrive at time $t_{1}(x)=x / D$; it means that the curve over $(-\infty, 0)$ will not reach extreme value and the curve over $(0,+\infty)$ must be an extremum at some time. The concave-convex of curve for given $x$ will change twice at $t_{2}(x)=x / D+(1 / D) \ln ((\sqrt{6}-\sqrt{2}) / 2)$ and $t_{3}(x)=$ $x / D+(1 / D) \ln ((\sqrt{6}+\sqrt{2}) / 2)$, respectively, where the interval of two times is invariant for every $x \geq \ln ((\sqrt{6}-\sqrt{2}) / 2)$. If $x<\ln ((\sqrt{6}-\sqrt{2}) / 2)$, the change does not happen.

Lemma 1. Suppose $0<D^{3}<3 / 8$ and $A(x)=4-12 x-$ $72 D^{3} x^{2}+216 D^{3} x^{3}-135 D^{3} x^{4}$, then there exists a unique $\zeta \in$ $(0,1)$, such that $A(\zeta)=0$.

Proof. It is clear that $A(0)=4$ and $A(1)=-8+9 D^{3}<0$, so we get $\zeta \in(0,1)$ which satisfies $A(\zeta)=0$.

$$
\begin{aligned}
& A^{\prime}(x)=-12-144 D^{3} x+648 D^{3} x^{2}-540 D^{3} x^{3}, \\
& A^{\prime \prime}(x)=4 D^{3}\left(-36+324 x-405 x^{2}\right) .
\end{aligned}
$$

We know that $A^{\prime}(x)$ arrived maximum at $x_{0}=(135+$ $\sqrt{8914}) / 405$ and $A^{\prime}\left(x_{0}\right)<32 D^{3}-12<0$; it indicates that $A^{\prime}\left(x_{0}\right)<0$ and $\zeta$ is unique. Thus the proof of Lemma 1 is completed.

Without loss of generality, we can assume that $\zeta=q / p$, where $p>q>0$ and they are relatively prime. It means $\operatorname{sech}^{2} \xi=\zeta=q / p$, then $\xi_{1}=\ln ((\sqrt{p}-\sqrt{p-q}) / \sqrt{q})$ and $\xi_{2}=\ln ((\sqrt{p}+\sqrt{p-q}) / \sqrt{q})$ solve the equation. By direct computation, we obtain that $\xi_{1}<0$ and $\xi_{2}>0$. The evolution procedure of the curvature at given $x$ can be described as follows.

Theorem 2. Suppose $0<D^{3}<3 / 8, K(t, x)$ is given by (18), and then

(a) if $0<x<(2 / \sqrt{D}) \xi_{1}$, the curvature $K(t, x)$ decrease with respect to $t$;

(b) if $(2 / \sqrt{D}) \xi_{1}<x<0$, the curvature $K(t, x)$ increase when $0<t<(1 / D)\left(x-(2 / \sqrt{D}) \xi_{1}\right)$ and decrease if $t>(1 / D)\left(x-(2 / \sqrt{D}) \xi_{1}\right)$ with respect to $t$; (c) if $0<x<(2 / \sqrt{D}) \xi_{2}$, the curvature $K(t, x)$ decrease when $0<t<x / D$; increase when $x / D<t<$ (1) $D)\left(x-(2 / \sqrt{D}) \xi_{1}\right)$; and decrease again when $t>(1 /$ D) $\left(x-(2 / \sqrt{D}) \xi_{1}\right)$;

(d) if $x>(2 / \sqrt{D}) \xi_{2}$, the curvature $K(t, x)$ increase when $0<t<(1 / D)\left(x-(2 / \sqrt{D}) \xi_{2}\right)$; decrease when $(1 /$ D) $\left(x-(2 / \sqrt{D}) \xi_{2}\right)<t<x / D$ and increase again if $x /$ $D<t<(1 / D)\left(x-(2 / \sqrt{D}) \xi_{1}\right)$; decrease again when $t>(1 / D)\left(x-(2 / \sqrt{D}) \xi_{1}\right)$.

For more perceptual intuition, we choose some different value for $x$ to show above properties via graph; one can see Figure 1.

On the other hand, we can obtain the description for evolution procedure of cumulative curvature at given $x$. If $x \in(-\infty,(2 / \sqrt{D}) \ln ((\sqrt{6}-\sqrt{2}) / 2))$, the cumulative curvature $I(t, x)$ is always decreased with respect to time $t$; if $x \in$ $((2 / \sqrt{D}) \ln ((\sqrt{6}-\sqrt{2}) / 2),(2 / \sqrt{D}) \ln ((\sqrt{6}+\sqrt{2}) / 2)), I(t, x)$ increase when $t<(1 / D)(x-(2 / \sqrt{D}) \ln ((\sqrt{6}-\sqrt{2}) / 2))$ and decrease when $t>(1 / D)(x-(2 / \sqrt{D}) \ln ((\sqrt{6}-\sqrt{2}) / 2))$. If $x \in((2 / \sqrt{D}) \ln ((\sqrt{6}+\sqrt{2}) / 2),+\infty), I(t, x)$ decrease when $t<(1 / D)(x-(2 / \sqrt{D}) \ln ((\sqrt{6}+\sqrt{2}) / 2))$ and increase when $(1 / D)(x-(2 / \sqrt{D}) \ln ((\sqrt{6}+\sqrt{2}) / 2))<t<(1 / D)(x-$ $(2 / \sqrt{D}) \ln ((\sqrt{6}-\sqrt{2}) / 2))$; meanwhile $I(t, x)$ decrease again when $t>(1 / D)(x-(2 / \sqrt{D}) \ln ((\sqrt{6}-\sqrt{2}) / 2))$. For $I(t, x)$, the changes of monotone indicate the orientation of curve bending of some segment of $\Gamma_{t}$ changing at time $t$; it coincides with the existence of inflection point.

Thus, for any given $x>0$, the curve $\Gamma_{t}(x)$ always has extreme value at $t^{*}=x / D$, but it is not true for any given $x<0$.

3.2. Modified KdV Equation. From (8), we obtain that

$$
u_{x}(t, x)= \pm \frac{D}{2} \operatorname{sech}^{2} \xi
$$

where $\xi=\sqrt{D / 2}(x+D t)$, with $D>0$ being a constant.

By differential (21) with respect to $t$,

$$
u_{x t}(t, x)=\mp D^{2} \sqrt{\frac{D}{2}} \operatorname{sech}^{2} \xi \tanh \xi .
$$


By differential (21) with respect to $x$,

$$
u_{x x}(t, x)=\mp D \sqrt{\frac{D}{2}} \operatorname{sech}^{2} \xi \tanh \xi
$$

Denoting

$$
g=\left(1+\frac{D^{4}}{4} \operatorname{sech}^{4} \xi\right)^{1 / 2}
$$

direct computation yields

$$
\begin{aligned}
K(t, x) & =\mp \frac{D}{g^{3}} \sqrt{\frac{D}{2}} \operatorname{sech}^{2} \xi \tanh \xi \\
I(t, x) & = \pm \frac{D}{2 g} \operatorname{sech}^{2} \xi .
\end{aligned}
$$

By differential (25) and (26) with respect to $t$, respectively, we obtain

$$
\frac{d K(t, x)}{d t}=\mp \frac{D^{3}}{8 g^{5}} B(\xi) \operatorname{sech}^{2} \xi,
$$

where $B(\xi)=D^{2} \operatorname{sech}^{4} \xi+12 \operatorname{sech}^{2} \xi-8$.

$$
\frac{d I(t, x)}{d t}=\mp \frac{D^{2}}{g^{3}} \sqrt{\frac{D}{2}} \operatorname{sech}^{2} \xi \tanh \xi .
$$

It is clear that the curve $\Gamma_{t}$ is always monotone, increasing, or decreasing which is depending on choosing sign "+" or "-." Without loose of generality, we just consider it in case of sign "+" in the remainder of this subsection. The inflection point of curve $\Gamma_{t}$ develops to $(-D t, 0)$ from $(0,0)$ and there is no extremum.

Lemma 3. Suppose that $B(x)=D^{2} x^{2}+12 x-8$, then there is a unique $x_{0} \in(0,1)$ such that $B\left(x_{0}\right)=0$.

Proof. By solving equation straightly,

$$
B(x)=D^{2} x^{2}+12 x-8=0,
$$

we have

$$
x_{0}=\frac{\sqrt{36+8 D^{2}}-6}{D^{2}}
$$

which belongs to $(0,1)$.

Thus, Lemma 3 is proved.

For convenience, we denote $x_{0}$ by $q / p$, where $p>q>0$ are constants depending on $D$. Then the evolution procedure of the curvature at given $x$ can be described as follows.

Theorem 4. The curvature $K(t, x)$ is given by (25), then

(a) if

$$
x>\sqrt{\frac{2}{D}} \ln \frac{\sqrt{p}+\sqrt{p-q}}{\sqrt{q}},
$$

then $K(t, x)$ always increase with respect to $t$ for any $t>0$;

(b) if

$\sqrt{\frac{2}{D}} \ln \frac{\sqrt{p}-\sqrt{p-q}}{\sqrt{q}}<x<\sqrt{\frac{2}{D}} \ln \frac{\sqrt{p}+\sqrt{p-q}}{\sqrt{q}}$,

then $K(t, x)$ decrease when $t<(1 / D) \sqrt{2 / D} \ln ((\sqrt{p}+$ $\sqrt{p-q}) / \sqrt{q})-x / D$ and increase when $t>(1 /$ D) $\sqrt{2 / D} \ln ((\sqrt{p}+\sqrt{p-q}) / \sqrt{q})-x / D$;

(c) if

$$
x<\sqrt{\frac{2}{D}} \ln \frac{\sqrt{p}-\sqrt{p-q}}{\sqrt{q}},
$$

then $K(t, x)$ increase when $t<(1 / D) \sqrt{2 / D} \ln ((\sqrt{p}-$ $\sqrt{p-q}) /-\sqrt{q})-x / D$ and decrease when (1/ D) $\sqrt{2 / D} \ln ((\sqrt{p}-\sqrt{p-q}) /-\sqrt{q})-x / D<t<(1 /$ D) $\sqrt{2 / D} \ln ((\sqrt{p}+\sqrt{p-q}) / \sqrt{q})-x / D$ and increase again when $t>(1 / D) \sqrt{2 / D} \ln ((\sqrt{p}+\sqrt{p-q}) / \sqrt{q})-$ $x / D$.

Theorem 5. $I(t, x)$ is given by (26), then

(a) if $x>0, I(t, x)$ always decrease with respect to $t$;

(b) if $x<0, I(t, x)$ increase when $t<-x / D$ and decrease when $t>-x / D$

There is no extremum for $\Gamma_{t}(x)$ which is regarded either as a curve of $t$ for given $x$ or as a curve of $x$ for fixed $t$.

3.3. Sine-Gordon Equation. By differential (10) with respect to $x$, we get

$$
u_{x}(t, x)=-\frac{\sqrt{1-D^{2}}}{(x-D t)^{2}+1-D^{2}},
$$

and then by direct computation, we have

$$
\begin{aligned}
& u_{x t}(t, x)=-\frac{2 D \sqrt{1-D^{2}}(x-D t)}{\left[(x-D t)^{2}+1-D^{2}\right]^{2}}, \\
& u_{x x}(t, x)=\frac{2 \sqrt{1-D^{2}}(x-D t)}{\left[(x-D t)^{2}+1-D^{2}\right]^{2}}, \\
& u_{x x t}(t, x)=\frac{2 D \sqrt{1-D^{2}}\left[(x-D t)^{2}+1-D^{2}\right]}{\left[(x-D t)^{2}+1-D^{2}\right]^{3}} .
\end{aligned}
$$

Let $g=\left(1+\left(1-D^{2}\right) /\left((x-D t)^{2}+1-D^{2}\right)^{2}\right)^{1 / 2}>1$; direct computation yields

$$
\begin{gathered}
K(t, x)=\frac{2 \sqrt{1-D^{2}}(x-D t)}{g^{3}\left[(x-D t)^{2}+1-D^{2}\right]^{2}}, \\
I(t, x)=-\frac{\sqrt{1-D^{2}}}{g\left[(x-D t)^{2}+1-D^{2}\right]} .
\end{gathered}
$$


By differential (36) and (37) with respect to $t$,

$$
\frac{d K(t, x)}{d t}=\frac{2 D \sqrt{1-D^{2}}}{g^{5}\left[(x-D t)^{2}+1-D^{2}\right]^{5}} C(x-D t),
$$

where

$$
\begin{aligned}
C(x-D t)= & (x-D t)^{6}+3\left(1-D^{2}\right)(x-D t)^{4} \\
& +3\left(1-D^{2}\right)^{2}(x-D t)^{2} \\
& -5\left(1-D^{2}\right)(x-D t)^{2} \\
& +\left(1-D^{2}\right)^{2}\left(2-D^{2}\right) \\
\frac{d I(t, x)}{d t}= & -\frac{2 D \sqrt{1-D^{2}}(x-D t)}{g^{3}\left[(x-D t)^{2}+1-D^{2}\right]^{2}}
\end{aligned}
$$

Lemma 6. Suppose that $D^{2} \leq 3 / 4$ and $C(x)=(x)^{3}+3(1-$ $\left.D^{2}\right)(x)^{2}+3\left(1-D^{2}\right)^{2}(x)-5\left(1-D^{2}\right)(x)+\left(1-D^{2}\right)^{2}\left(2-D^{2}\right)$, then there exist $0<x_{1}<x_{2}$, such that $C\left(x_{1}\right)=C\left(x_{2}\right)=0$.

Proof. By differential $C(x)$ with respect to $x$, we can get

$$
C^{\prime}(x)=3 x^{2}+6\left(1-D^{2}\right) x-\left(1-D^{2}\right)\left(3 D^{2}+2\right) .
$$

Solving equation $C^{\prime}(x)=0$, we obtain a stationary point $x_{0}=\left(\sqrt{15\left(1-D^{2}\right)}-3\left(1-D^{2}\right)\right) / 3>0$. Throughout rigmarole computing, we obtain $\min C(x)=C\left(x_{0}\right)$, and

$$
\begin{aligned}
C\left(x_{0}\right)= & -\frac{\sqrt{15}}{9}\left(1-D^{2}\right)^{3 / 2}+3\left(1-D^{2}\right)^{2} \\
& -\frac{5 \sqrt{15}}{3}\left(1-D^{2}\right)^{5 / 2}+3\left(1-D^{2}\right)^{3}<0 .
\end{aligned}
$$

Noticing that $C(0)>0$ and $C(x)>0$ hold true for large enough $x$, according to calculus basic theory, we assert that there is $0<x_{1}<x_{2}$ which satisfies, respectively, $C\left(x_{1}\right)=$ $C\left(x_{2}\right)=0$. Then lemma is proved.

From (38) and Lemma 3, we derive the following results.

Theorem 7. Suppose $D^{2}<3 / 4, x_{1}$, and $x_{2}$ are given by Lemma 3, then

(a) if $x<-\sqrt{x_{2}}, K(t, x)$ defined by (36) always increase with respect to $t$;

(b) if $-\sqrt{x_{2}}<x<-\sqrt{x_{1}}, K(t, x)$ decrease when $t<(x+$ $\left.\sqrt{x_{2}}\right) / D$ and increase when $t>\left(x+\sqrt{x_{2}}\right) / D$;

(c) if $-\sqrt{x_{1}}<x<\sqrt{x_{1}}, K(t, x)$ increase when $t<$ $\left(x+\sqrt{x_{1}}\right) / D$, decrease when $\left(x+\sqrt{x_{1}}\right) / D<t<$ $\left(x+\sqrt{x_{2}}\right) / D$, and increase again when $t>\left(x+\sqrt{x_{2}}\right) / D$;

(d) if $\sqrt{x_{1}}<x<\sqrt{x_{2}}, K(t, x)$ decrease when $t<$ $\left(x-\sqrt{x_{1}}\right) / D$, increase when $\left(x-\sqrt{x_{1}}\right) / D<t<$ $\left(x+\sqrt{x_{1}}\right) / D$, decrease again when $\left(x+\sqrt{x_{1}}\right) / D<$ $t<\left(x+\sqrt{x_{2}}\right) / D$, and finally increase again when $t>\left(x+\sqrt{x_{2}}\right) / D$; (e) if $x>\sqrt{x_{2}}, K(t, x)$ increase when $t<\left(x-\sqrt{x_{2}}\right) / D$, decrease when $\left(x-\sqrt{x_{2}}\right) / D<t<\left(x-\sqrt{x_{1}}\right) / D$, increase when $\left(x-\sqrt{x_{1}}\right) / D<t<\left(x+\sqrt{x_{1}}\right) / D$, decrease again when $\left(x+\sqrt{x_{1}}\right) / D<t<\left(x+\sqrt{x_{2}}\right) / D$, and finally increase again when $t>\left(x+\sqrt{x_{2}}\right) / D$.

Theorem 8. Assume that $I(t, x)$ is given by (37), then

(a) if $x<0, I(t, x)$ always increase with respect to time $t$;

(b) if $x>0, I(t, x)$ decrease when $t<x / D$ and increase when $t>x / D$.

There is no extremum for $\Gamma_{t}(x)$ which is regarded either as a curve of $t$ for given $x$ or as a curve of $x$ for fixed $t$.

3.4. Camassa-Holm Equation. By differential (12) with respect to $x$, we get

$$
u_{x}(t, x)=-c e^{-|x-c t|} \operatorname{sgn}(x-c t),
$$

and then by direct computation, we have

$$
\begin{aligned}
& u_{x x}(t, x)=-c e^{-|x-c t|} \\
& u_{x t}(t, x)=-c^{2} e^{-|x-c t|}
\end{aligned}
$$

Let

$$
g=\left(1+c^{2} e^{-2|x-c t|}\right)^{1 / 2}
$$

we have

$$
\begin{aligned}
K(t, x) & =\frac{c}{g^{3}} e^{-|x-c t|}, \\
I(t, x) & =\frac{c}{g} e^{-|x-c t|} \operatorname{sgn}(x-c t) .
\end{aligned}
$$

By differential (45) and (46) with respect to $t$, we get

$$
\begin{aligned}
& \frac{d K(t, x)}{d t}=\frac{c^{2}}{g^{5}} \operatorname{sgn}(x-c t) e^{-|x-c t|}\left(1-2 c^{2} e^{-2|x-c t|}\right), \\
& \frac{d I(t, x)}{d t}=-\frac{c^{2}}{g^{3}} e^{-|x-c t|} .
\end{aligned}
$$

It is clear that the extremum point of the curve $\Gamma_{t}$ which is defined by (12) develops to $c t$ from 0 , and there is no inflection point for the curve $\Gamma_{t}$.

Theorem 9. Suppose that $K(t, x)$ is given by (45), then

(a) if $x<-(1 / 2) \ln 2 c^{2}, K(t, x)$ always decrease with respect to time t for any $t>0$;

(b) if $-(1 / 2) \ln 2 c^{2}<x<0, K(t, x)$ increase when $t<$ $x / c+\ln 2 c^{2} / 2 c$ and decrease when $t>x / c+\ln 2 c^{2} / 2 c ;$

(c) if $0<x<(1 / 2) \ln 2 c^{2}, K(t, x)$ decrease when $t<x / c$, increase when $x / c<t<x / c+\ln 2 c^{2} / 2 c$, and finally decrease again when $t>x / c+\ln 2 c^{2} / 2 c$; 
(d) if $x>(1 / 2) \ln 2 c^{2}, K(t, x)$ increase when $t<x / c-$ $\ln 2 c^{2} / 2 c$, decrease when $x / c-\ln 2 c^{2} / 2 c<t<x / c$, increase again when $x / c<t<x / c+\ln 2 c^{2} / 2 c$, and finally decrease again when $t>x / c+\ln 2 c^{2} / 2 c$.

From (48), we obtain that $I(t, x)$ given by (46) always decrease with respect to time $t$ for any $t>0$.

For any given $x>0$, the curve $\Gamma_{t}$ always has extreme value at $t^{*}=x / D$, but it is not true for any given $x<0$.

\section{Conclusion}

From the above discussion, the behavior of the curve group $\Gamma_{t}$ as a function of $t$ for any given $x$ is different with that as a function of $x$ for any given $t$. The evolution procedure of curvature which is given by (6) and (12), respectively, is similar, but it is different to those curves given by (8) and (10). Even though, for self-similar curve group, different points $(x, u(x, t))$ possess different evolution procedures of curvature, all evolution results are the same. For some points, the curvature of $\Gamma_{t}(x)$ uniformly monotonously tends to final state when $t$ goes to infinity, but for other points, the curvature of $\Gamma_{t}(x)$ tends to final state after several fluctuations. Besides this, this paper investigates fluctuation for every given $x$ with respect to time $t$. We find that, for some $x, u(x, t)$ will have its extreme value at some $t^{*}$, but for another case, it never can happen. It is an interesting phenomenon.

\section{Conflicts of Interest}

The authors declare that they have no conflicts of interest.

\section{Acknowledgments}

This research was supported by the National Nature Science Foundation of China (Grants nos. 11371175, 11501253, 11571141, and 11571140), the Nature Science Foundation of Jiangsu Province (Grant no. BK20140525), and the Advanced Talent of Jiangsu University (Grants nos. 14JDG070 and 15JDG079).

\section{References}

[1] R. Camassa and D. D. Holm, "An integrable shallow water equation with peaked solitons," Physical Review Letters, vol. 71, no. 11, pp. 1661-1664, 1993.

[2] A. Constantin and W. A. Strauss, "Stability of peakons," Communications on Pure and Applied Mathematics, vol. 53, no. 5, pp. 603-610, 2000

[3] J. Lenells, "Traveling wave solutions of the Camassa-Holm equation," Journal of Differential Equations, vol. 217, no. 2, pp. 393-430, 2005.

[4] A. C. Newell, Solitons in Mathematics and Physics, SIAM, Philadelphia, Pa, USA, 1985.

[5] R. S. Johnson, "Camassa-Holm, Korteweg-de Vries and related models for water waves," Journal of Fluid Mechanics, vol. 455, pp. 63-82, 2002.

[6] D.-X. Kong and Z.-G. Wang, "Formation of singularities in the motion of plane curves under hyperbolic mean curvature flow,"
Journal of Differential Equations, vol. 247, no. 6, pp. 1694-1719, 2009.

[7] M. Nara and M. Taniguchi, "The condition on the stability of stationary lines in a curvature flow in the whole plane," Journal of Differential Equations, vol. 237, no. 1, pp. 61-76, 2007.

[8] H.-Y. Jian and H.-J. Ju, "Existence of translating solutions to the flow by powers of mean curvature on unbounded domains," Journal of Differential Equations, vol. 250, no. 10, pp. 3967-3987, 2011.

[9] G. Bellettini, V. Caselles, A. Chambolle, and M. Novaga, "The volume preserving crystalline mean curvature flow of convex sets in RN," Journal de Mathématiques Pures et Appliquées, vol. 92, no. 5, pp. 499-527, 2009.

[10] M. Craizer and R. Teixeira, "Evolution of an extremum by curvature motion," Journal of Mathematical Analysis and Applications, vol. 293, no. 2, pp. 721-737, 2004.

[11] E. L. Mansfield and P. H. van der Kamp, "Evolution of curvature invariants and lifting integrability," Journal of Geometry and Physics, vol. 56, no. 8, pp. 1294-1325, 2006.

[12] J. S. Langer, "Instabilities and pattern formation in crystal growth," Reviews of Modern Physics, vol. 52, no. 1, pp. 1-28, 1980.

[13] E. Ben-Jacob, N. Goldenfeld, J. S. Langer, and G. Schön, "Dynamics of Interfacial Pattern Formation," Physical Review Letters, vol. 51, no. 21, pp. 1930-1932, 1983.

[14] J. A. Sethian, "Curvature and the evolution of fronts," Communications in Mathematical Physics, vol. 101, no. 4, pp. 487-499, 1985.

[15] S. Osher and J. A. Sethian, "Fronts propagating with curvaturedependent speed: algorithms based on Hamilton-Jacobi formulations," Journal of Computational Physics, vol. 79, no. 1, pp. 1249, 1988.

[16] M. Gage and R. S. Hamilton, "The heat equation shrinking convex plane curves," Journal of Differential Geometry, vol. 23, no. 1, pp. 69-96, 1986.

[17] M. A. Grayson, "The heat equation shrinks embedded plane curves to round points," Journal of Differential Geometry, vol. 26, no. 2, pp. 285-314, 1987.

[18] B. B. Kimia, A. Tannenbaum, and S. . Zucker, "On the evolution of curves via a function of curvature. I. The classical case," Journal of Mathematical Analysis and Applications, vol. 163, no. 2, pp. 438-458, 1992.

[19] M. J. Ablowitz, B. M. Herbst, and C. Schober, "On the numerical solution of the sine-Gordon equation. I. INTegrable discretizations and homoclinic manifolds," Journal of Computational Physics, vol. 126, no. 2, pp. 299-314, 1996. 


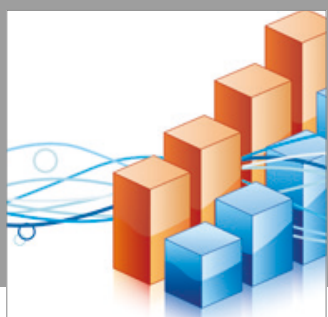

Advances in

Operations Research

vatersals

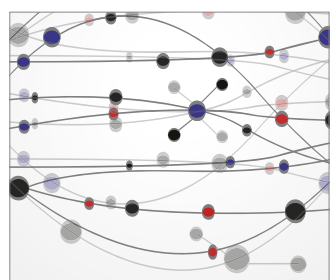

\section{The Scientific} World Journal
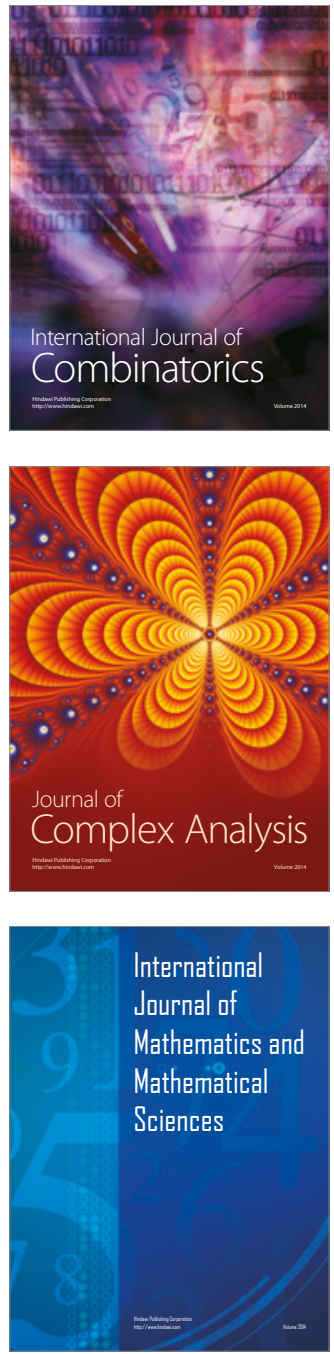
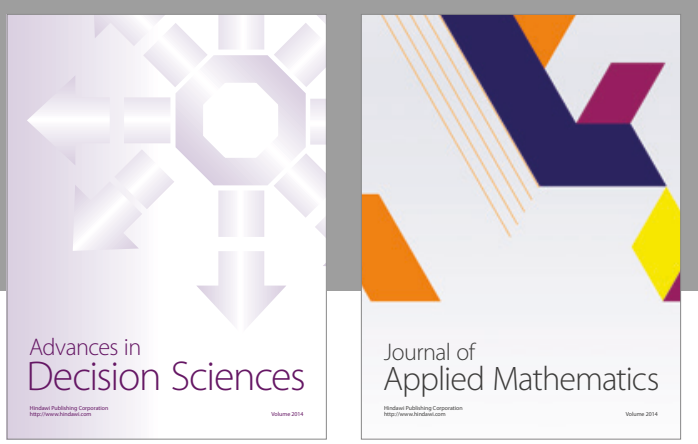

Algebra

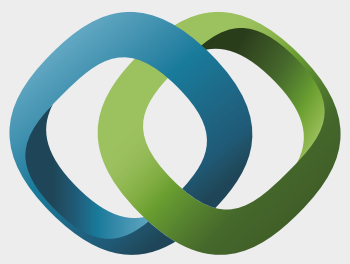

\section{Hindawi}

Submit your manuscripts at

https://www.hindawi.com
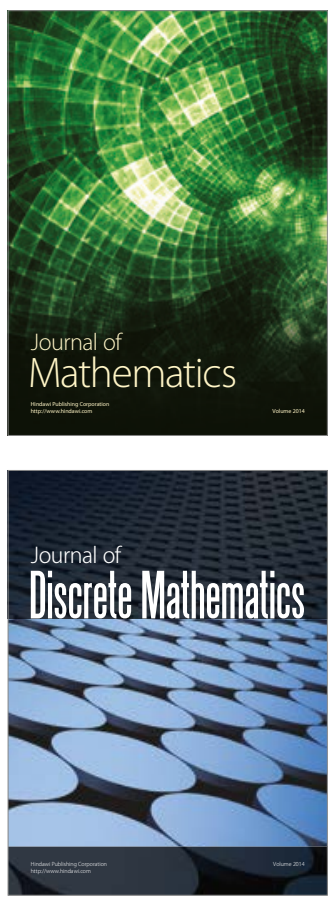

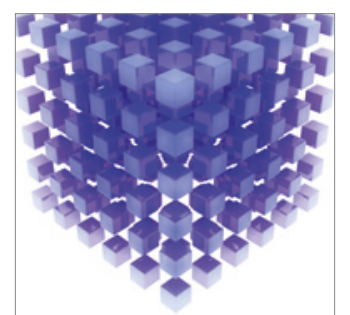

Mathematical Problems in Engineering
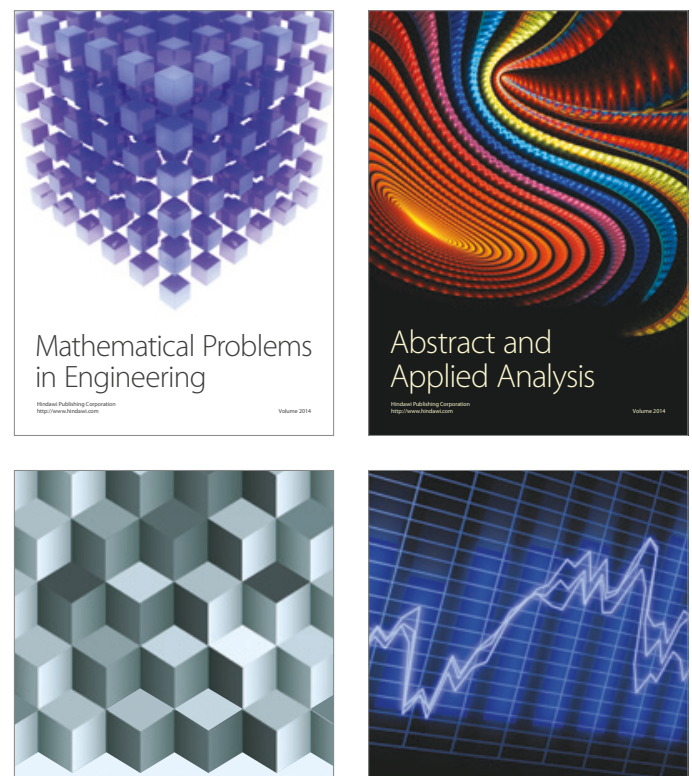

Journal of

Function Spaces

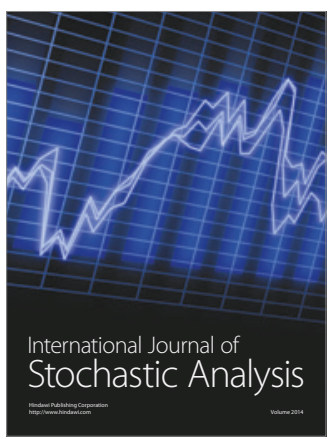

Probability and Statistics
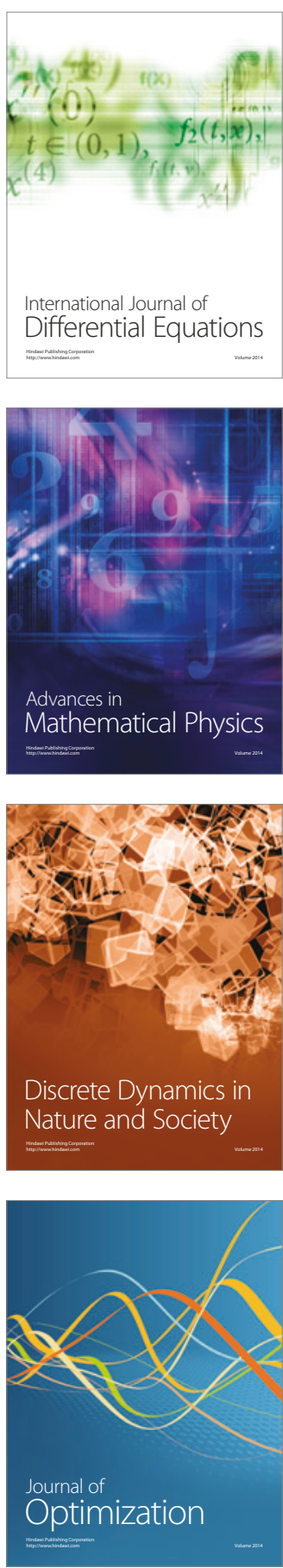